\title{
diseño de proyectos como herramienta de trabajo del gestor cultural
}

\author{
"A Alfons, con afecto, por todo aquello de aquí \\ que también es suyo"
}

\section{Resumen}

El diseño de los proyectos es una herramienta de trabajo importante para los profesionales que trabajan en la gestión de los diferentes sectores de la cultura. Permite estructurar el trabajo, conocer bien el contexto en el que se va a intervenir, tomar las decisiones lo más acorde posible con la realidad y los medios disponibles, organizar todo el proceso de producción, evaluar nuestras acciones, etc. En una situación permanente de escasez relativa de recursos el diseño de los proyectos facilita obtener el máximo de resultados acertados con el mínimo de costos en clave de medios empleados. Lo que aquí se expone desarrolla y amplía esta línea argumental.

\section{Palabras clave}

Proyecto / Contexto / Objetivo / Estrategia / Evaluación

\section{Qué es un proyecto y para qué sirve}

En la vida cotidiana hacemos constantemente proyectos. Cada una de nuestras decisiones requiere de una estructura mental para llevarla a cabo. Si preparamos las vacaciones para este verano tendremos que plantearnos que queremos lograr, nos informaremos de como es el lugar al que queremos ir, las diferentes maneras de viajar por él, decidiremos un itinerario y unos lugares que visitar y haremos todo lo posible para planificar su preparación, calcular un presupuesto aproximado, etc. Lo mismo pasa si planeamos cambiar de trabajo, tener un hijo o preparar la cena de esta noche.
Esto, que hacemos de manera más o menos intuitiva según el caso, es el desarrollo natural de un proyecto con sus etapas, su aplicación y su evaluación. Si ya lo hacemos cada día, sólo nos falta estructurarlo mejor para los proyectos profesionales que pueden ser menos repetitivos en el tiempo y más complejos por su tamaño, compromisos adquiridos, recursos invertidos y personas involucradas.

El proyecto es pues la concreción de una voluntad a la que llamamos política cultural, entendiendo por esta el conjunto de valores, ideas y voluntades que una organización quiere desarrollar. Para ello es necesario "traducir" las ideas a acciones concretas que deben estructurarse de la mejor manera posible para lograr, con la máxima eficiencia, la finalidad planteada. Visto así, un proyecto necesita de una política cultural establecida previamente. Cuando el proyecto no responde a esta voluntad previa, ya sea porque hay un desajuste entre ambos ya sea porqué la política cultural no existe o no está bien definida, el proyecto es un simple acto mecánico con un muy elevado grado de duda en cuanto a su idoneidad. Se hace por hacer sin saber a qué corresponde tanto activismo.

El diseño de proyectos se presenta entonces como la herramienta de trabajo del gestor cultural que utiliza para desarrollar las ideas expuestas y concretarlas en acciones. Debe entenderse además que el proyecto es un instrumento colectivo y compartido, que se domina como el carpintero maneja la sierra o el martillo y que debe saberse usar para el buen desarrollo de las acciones sin que se convierta en un fin por ella misma.

Otras profesiones también trabajan con proyectos. ¿Sería aceptable que un arquitecto quisiera construir una casa sin un estudio y unos planos previos? ¿Son los planos un simple requerimiento formal para pedir el permiso de obras, que luego se olvida o es, en cambio, la garantía que se tiene de que la construcción se desarrollará de acuerdo con unos mínimos de previsión, seguridad, calidad, etc.? Y así podemos decir lo mismo de un médico antes de intervenir un paciente o de un maestro que orienta su curso escolar.

\section{David Roselló Cerezuela}

Gestor cultural 
En el campo cultural, se trabaja con el mismo método para pequeños y grandes proyectos. En todos ellos existen las mismas fases. La diferencia está en que los pequeños proyectos se desarrollan de una manera más mecánica y espontánea porqué se suele conocer bien sus condiciones, y en los grandes se incrementa la cautela y se les da respuestas más previsoras, calculadas, etc.

\section{Tres criterios básicos a la hora de trabajar por proyectos}

En el trabajo por proyectos se nos presentan, al menos, los siguientes criterios básicos:

- El martillo

Los gestores culturales deben asumir el trabajo por proyectos como su herramienta de trabajo útil para garantizar el buen fin de las acciones. Así, el proyecto es su elemento de referencia durante todo el proceso y se utiliza para mejorar el trabajo. Sin él se trabajaría mucho peor.

- El librillo del maestrillo

No hay un esquema único válido para todos los proyectos, todas las personas, todos los temas, etc. Los esquemas planteados deben verse como una guía que ayuda a recorrer el camino metodológico pero que debe dejar margen en su aplicación. Así el diseño de un proyecto puede variar según el equipo de gestores culturales, el cliente que lo va a recibir, el tema a tratar, la amplitud y envergadura, la novedad, etc.

- La película

El orden de filmación de las diferentes escenas de una película no es forzosamente el mismo en que se visualizan. De la misma manera, aunque el orden de lectura de los capítulos de un proyecto sea uno, este no se corresponde al orden en que fueron redactados. A menudo se empieza a redactar un proyecto por un apartado, luego se empieza otro capítulo, se retorna al anterior, se salta varios aspectos hasta que a medida que avanza su diseño, se van completando sus diferentes partes.

\section{Definición terminológica}

Conviene aquí hacer un pequeño repaso de los conceptos más utilizados habitualmente en el sector profesional.

Plan: Es el conjunto de grandes líneas directrices que orientan una voluntad de intervención. Se plantea a nivel general, de amplia base conceptual, filosófica o política. Un plan suele trazarse a mediano o largo plazo y acostumbra a abarcar un amplio conjunto de temas o aspectos incluidos en él.

Ej.: Plan estratégico del sector cultural de una ciudad, el plan de bibliotecas, etc.

Programa: Es una primera concreción del plan. Recoge las líneas directrices y las aplica a un aspecto concreto, por sectores (ej.: programa de artes escénicas), por edades (ej.: programa de juventud), por funciones (ej.: programa de apoyo a la creación), por espacios (ej.: programa de un equipamiento), etc.

Proyecto: Es la línea más operativa de una intervención. Desarrolla los programas y los materializa en acciones concretas que se agrupan bajo el nombre de proyecto. Es la unidad mínima de actuación con sentido en si misma. Se concreta en actividades o acciones que sólo tienen razón de ser en cuanto forman parte del proyecto. (Ej.: Un ciclo estable de teatro, un festival de cine, la fiesta mayor, un taller, etc.)

Ejemplo global: Una institución se plantea el siguiente esquema de trabajo.

Plan: desarrollo de las artes en el territorio de incidencia. Programa: potenciación de la danza

Proyecto: actuaciones en la calle para celebrar del día internacional de la danza.

De la misma manera que aquí se presenta el orden "programa-proyecto" hay quien los utiliza invertidos "proyecto-programa". Siendo ambas posibilidades válidas lo importante es saber que nuestras acciones pueden desarrollarse en diferentes niveles de concreción. Este texto toma el proyecto como el último nivel de concreción.

Si se miran los verbos asociados, se ve que no son útiles en el mismo sentido.

- Planear apenas se usa en cultura. Es más corriente en la planificación estratégica como sustantivo: planeamiento.

- Programar, lejos de ser el establecimiento de programas, se entiende por realizar la programación de actividades.

- Proyectar no se usa.

- Planificar, en cambio, junto con diseñar es el término más usado en la estructuración de proyectos culturales.

\section{Justificación del trabajo por proyectos}

Varias son las razones que justifican la necesidad o la utilidad de establecer un sistema estructurado para desarrollar el trabajo cotidiano de un gestor cultural. He aquí algunas respuestas a esta inquietud. El diseño de los proyectos culturales permite:

- Optimizar recursos para conseguir máximos resultados.

En una situación (habitual en la profesión) en la que se exige obtener muchos y buenos resultados pero contando con recursos a menudo bastante limitados, se necesita una herramienta de planificación que permita buscar una relación lo más ventajosa posible. Se puede así aprovechar mejor los recursos de todo tipo (humanos, económicos, materiales, etc.) y utilizarlos lo mejor posible para conseguir los objetivos deseados. 
- Orientar las acciones desde el principio. Se trata de evitar el método de ensayo y error tan propio de otras disciplinas como por ejemplo la investigación científica en la que, al no lograr resultados en una línea de trabajo, la abandona e inicia otra. En cultura se tiene suficientes elementos de certeza como para no "probar" demasiado un programa, una actividad o una línea de trabajo. Aunque siempre hay un margen de duda y riesgo, también hay, si se trabaja bien, maneras de acercarse al conocimiento de lo que puede suceder.

- Romper viejos esquemas de planificación.

En estructuras que llevan mucho tiempo trabajando de la misma manera, el diseño de los proyectos puede aportar una renovación de los sistemas de planificación. En particular el hecho de planificar una intervención en equipo hace que todos y cada uno de sus componentes asuman su parte de responsabilidad en el trabajo. Ya no se trata de que la dirección del proyecto diseña hasta el último detalle y lo comunica a los ejecutores para que lo lleven a cabo como si fuera un manual de instrucciones. La participación de todo el equipo aporta, implicación, responsabilidad, ideas, coordinación, exigencia colectiva, etc. que redundan en una mejora del proyecto.

- Hacer un proceso de reflexión y relanzamiento de ideas.

Aunque un proyecto ya funcione, conviene actualizarlo permanentemente para no caer en el tentación de tomar el anterior como guía y cambiar sólo algunos aspectos muy particulares. Al contrario, un proyecto debería recibir una buena sacudida cada vez para ponerlo a prueba y justificar cada paso dado. La rutina en un proyecto es un mal que lo desmejora poco a poco pero efectivamente.

- Establecer prioridades, criterios y actitudes comunes. Un equipo, por muy compenetrado que esté o por mucha veteranía acumulada que tenga necesita tomar acuerdos compartidos, comunes a todos sus componentes e igualmente visibles de cara al exterior.

- Generalizar las prácticas de seguimiento y control. El hecho de diseñar un proyecto conlleva adquirir el hábito de hacer su seguimiento para comprobar si su desarrollo se ajusta a lo previsto. En el caso contrario, la no planificación se acompaña casi forzosamente de una falta de seguimiento y control.

- Disponer de un documento escrito para su presentación.

Un proyecto puede presentarse a alguien de manera oral, sin más. Pero de todos es sabido que un documento escrito, bien presentado en la forma así como convincente en su contenido, ayuda mucho a que sea entendido y aceptado por un interlocutor.

- Disponer de un documento escrito que perdura. En el sector cultural existe una cierta agrafía a la hora de escribir los proyectos o las reflexiones propias de la profesión. Cuando se buscan antecedentes, escritos diversos o simplemente proyectos anteriores hay una dificultad manifiesta en encontrarlos de manera escrita, completa y coherente. Como mucho se encuentran documentos parciales y no actualizados de intervenciones realizadas. Diseñar y escribir los proyectos es una buena manera de contribuir a que perduren incrementando el "poso" documental necesario a la consolidación tanto de los proyectos como de la profesión.

- Estimular y cohesionar a los profesionales.

Si a un equipo de profesionales cansados de trabajar de manera improvisada, de hoy para mañana, sin criterios de intervención, etc. se le propone trabajar de manera ordenada y metódica, pensando a largo plazo, con dimensión de globalidad, apelando a su profesionalidad, etc. se logrará su estímulo y la cohesión del equipo.

- Mostrar un método de trabajo a las personas que se incorporan.

Si existe un método de trabajo lógico y ordenado, con previsiones, prioridades, debate interno, seguimiento y control, etc. será más fácil la incorporación de personas nuevas en el equipo pues se acoplarán mejor al ritmo de trabajo del equipo. En el caso contrario, la duda y la incertidumbre descolocarán a la persona recién llegada al equipo.

- Movilizar diferentes agentes en un proyecto común. Trabajar de manera ordenada y eficiente ayuda a cooperar a diferentes personas e instituciones en un proyecto común. El debate y la toma de decisiones obligan a acoplar los ritmos de trabajo y las ideas. Ya no se trata de realizar un proyecto en solitario y presentarlo a los demás sino de hacerlo en común desde un inicio.

- Ofrecer una imagen de seriedad y profesionalidad. Es importante mostrar que se trabaja de manera profesional en el diseño de un proyecto cultural, que no se confía a la simple intuición de los hechos y la improvisación en las acciones. Un proyecto bien estructurado y presentado es esencial si queremos convencer a nuestros interlocutores.

\section{Requisitos del diseño de proyectos culturales}

¿Qué se le pide a un proyecto cultural, tanto en su diseño como en su resultado, para ser considerado un buen proyecto?

No cualquier documento es un buen proyecto y no nos referimos sólo a las ideas geniales o a una exhibición desorbitada de medios. Ni un buen proyecto es el más grueso ni el que se extiende más en el uso de términos complejos, tablas y gráficas impactantes o imágenes a todo color.

Al contrario, el diseño de un proyecto debe, ante todo, cumplir algunos, si no todos, los siguientes requisitos: 
- Permitir la toma de decisiones.

Un proyecto debe ser útil para quién lo maneja y para quién lo recibe. Es un texto claro y conciso que guía el equipo en su quehacer cotidiano, le sirve de herramienta útil, refleja la realidad del trabajo a realizar y plantea todos los elementos necesarios para los diferentes responsables. No es, por lo tanto, aquel documento bonito que se escribe pensando sólo en el interlocutor (la mayoría de los casos con el único fin de conseguir financiación) y que luego se guarda en el fondo del cajón porqué ya cumplió su función y el equipo se dispone a trabajar "a su manera".

- Tener perspectiva de globalidad.

Aunque la intervención se suscriba a un aspecto limitado en el tiempo, el espacio, los sectores, etc., el proyecto debe tener una perspectiva mucho más amplia teniendo en consideración el amplio contexto en el que se inscribe. Un proyecto, por pequeño que sea debe de estar bien ubicado.

- Ser coherente entre sus fases.

Esta buena relación con su entorno debe aplicarse también en su interior. Un proyecto no es una simple suma de diferentes partes. Es una totalidad coherente entre si en la que cada capítulo es el desarrollo de una idea central común. No funciona, por lo tanto, una redacción por capítulos que cada miembro del equipo se encarga de realizar y que luego alguien se encarga de juntar con más o menos gracia.

- Ser fruto de un proceso anterior y origen de uno posterior.

Tal vez no es una condición "sinequanon" pero es interesante que un proyecto sepa recoger ideas, iniciativas y acciones existentes del pasado y del presente. Se presenta así como una continuación si las mantiene o un cambio si rompe con ellas, pero en todo caso las tiene en cuenta y las valora. De la misma manera, los proyectos deben pensar en su continuidad desde un inicio. No se trata, una vez constatado el éxito del proyecto, de plantearse "algo" para darle continuidad. Puede ser demasiado tarde si no se ha trabajado el tema antes.

- Ser realista y consecuente con el contexto.

Casi es un axioma pero un proyecto no puede estar desligado de su contexto inmediato. Se debe acoplar a él, nace de él y representa una respuesta organizada a su realidad. Conocer a fondo los elementos más destacados de este entorno es esencial a la hora de diseñar cualquier propuesta de intervención.

- Estar abierto a la participación de otros agentes. Un proyecto debe facilitar la incorporación de otros agentes en su misma fase de diseño. Tiene que poder ampliar el debate, aceptar otros puntos de vista, etc.

- Conocer los presupuestos ideológicos que lo guían. Es esencial conocer la ideología de la institución titular del proyecto. Éste debe ser una respuesta en su misma línea de trabajo y pensamiento, al margen de las ideas de las personas redactoras del mismo.
Cuanto más coincidentes sean, más efectivo será el planteamiento y el desarrollo del proyecto.

- Establecer una temporalización del proyecto. El diseño del proyecto es una suma de ideas y acciones a desarrollar que requieren de una temporalización para ordenar el debate en el tiempo, facilitar la toma de decisiones y su ejecución, establecer fechas límite de las distintas fases para llegar bien a su realización.

\section{Los diferentes niveles de un proyecto}

¿Cuando un proyecto es ya un proyecto definitivo?

Existen diferentes niveles de un mismo proyecto que se suceden en el tiempo. Cada nivel representa una concreción y ampliación del anterior hasta llegar al más completo y definitivo de todos: el proyecto ya realizado.

La idea: Muchos proyectos nacen en una servilleta de papel con pocas ideas y algún esquema. Es el inicio de un proyecto. Puede incorporar ya el tema a tratar o las dudas a resolver. Identifica el punto de partida y le da sentido, aunque todavía quedan muchos aspectos por aclarar.

El primer esbozo: Si se da un paso más, la simple idea se transforma en un pequeño desarrollo en el se concretan de manera genérica todavía, algunas propuestas, algún dato relevante en cuanto a espacios, tiempo, personas, temas, etc. que se plantean de acuerdo a una realidad que todos comparten.

El anteproyecto: Si la cosa parece que va en serio, se pasa a desarrollar más cada punto. Aparecen los capítulos que conformarán el proyecto. Se analiza el contexto con más detalle. Se profundiza más en las propuestas, dejándolas casi definitivas pero no se entra en los detalles de la planificación, la gestión, la evaluación, etc. Está sujeto a variaciones según se desarrolle el debate y la aprobación.

El proyecto a ejecutar: Es lo más parecido a la realidad que se tiene justo antes de llevar a cabo el proyecto. Contiene todo el detalle necesario para su ejecución, tanto a nivel de las ideas, las acciones, la gestión, etc. Es como el manual de trabajo para el equipo y a él se remiten los profesionales para su desarrollo.

El proyecto realizado: Es el proyecto definitivo y es la resultante de la incorporación de todos los cambios habidos durante su realización. Ejerce de memoria en algunos casos. Significa el cierre definitivo del ciclo vital de todo proyecto cultural.

\section{Las diferentes versiones de un proyecto}

Existen diferentes versiones o presentaciones de un mismo proyecto. Según para qué lector esté pensado se debe elaborar una versión con aquello que más puede interesarle. 
A la dirección se les destacará los objetivos a corto y largo plazo, el impacto que supondrá para la institución, el uso de recursos internos, la relación con otros agentes, el presupuesto, etc.

A los medios de comunicación se les presentará aquellos aspectos del proyecto con más impacto mediático, las actividades a realizar, los nombres famosos, etc.

A los patrocinadores les interesará sobretodo su presencia en imagen, el tipo de público al que va dirigido el proyecto, la repercusión de su aportación, las instituciones convocantes, etc.

A los artistas se les mostrará el significado global del proyecto, su impacto en la población, el papel de su participación, los otros artistas implicados, las entidades convocantes, los datos concretos de su actuación, etc.

A la entidades y colectivos sociales les importará conocer la finalidad del proyecto, la repercusión en su territorio o sector de influencia, el papel de su participación, los otros agentes implicados, etc.

Pero el más importante de todos ellos es el proyecto que utiliza el equipo como instrumento de trabajo. Es el documento base, el más completo, el que incluye a todos los anteriores, el que vela para que las diferentes fases sean coherentes entre si. Los demás son versiones adaptadas para cada uso.

\section{Dificultades del diseño de proyectos culturales}

En el quehacer diario de un gestor cultural existen numerosas dificultades que limitan o impiden el pleno desarrollo del proceso de diseño de un proyecto tal como sería de desear. No por ello el profesional debe abandonarse a la fatalidad sino que debe intentar encontrar las soluciones en cada caso para acercarse lo más posible al desarrollo óptimo de su trabajo. Tampoco debe ver siempre el enemigo en su entorno (el jefe que no se entera, la falta constante de recursos, los encargos "para ayer", etc.) sino que debe hacer una autocrítica en aquello en que uno mismo está fallando.

Seguro que hay más ni todas lo son para todo el mundo, pero he aquí algunas de las dificultades que afronta el gestor cultural en el diseño de proyectos:

- No está suficientemente valorado ("pérdida de tiempo").

Ciertamente el hecho de dedicar todo un equipo de profesionales un tiempo a planificar los proyectos no goza siempre de la aprobación de los superiores. O bien se considera una pérdida de tiempo innecesaria o, aun aceptando su interés, no se encuentra nunca el momento propicio pues hay cosas más importantes o urgentes para realizar.

Diseñar convenientemente un proyecto se convierte en un lujo que se da uno cuando no tiene nada más que hacer o cuando todas las condiciones están a favor...cosa que nunca sucede.

- Se busca el cuestionario para resolver la falta de estructuración.

Sobretodo en las personas que tienen poca experiencia profesional, se da el caso de quién, por inseguridad o por comodidad busca un esquema de proyecto que le sirva de cuestionario al que sólo debe rellenar las respuestas a cada pregunta. Se ahorra así el esfuerzo y evita el riesgo de estructurar el proyecto, de seleccionar aquello que debería considerar como importante y lo que no, etc. Otra cosa es que algunas instituciones que otorgan ayudas económicas usen el sistema del cuestionario para homogeneizar el formato de las peticiones y facilitar su lectura. Pero, hay que recordar que cuando se habla de proyecto cultural se habla de la herramienta de trabajo del equipo de profesionales.

\section{- Complejo del misionero.}

Algunos profesionales tienen la sensación equivocada de ser los primeros en abordar un tema que nadie hasta ahora ha tratado. Consecuentemente, no buscarán experiencias previas que puedan darles alguna orientación útil. Por suerte, hoy en día, muchos campos de intervención de la gestión cultural han sido ya trabajados desde diferentes agentes, ópticas y proyectos. Aunque no siempre se encuentra referentes idénticos a los de uno mismo, hay suficiente experiencia acumulada para tomarla en cuenta.

\section{- Muro de las lamentaciones.}

Una característica propia de algunos profesionales es la de destacar ante todo las desgracias propias y no ver las potencialidades de la situación en la que se encuentra. Es común lamentarse de la falta de presupuesto, de medios humanos, de tiempo, de planes globales, de una dirección firme y conocedora, etc.

\section{- Proyectos "seta".}

¿Cuántas veces han aparecido proyectos de la noche a la mañana sin que nadie sepa de donde salen? Son intervenciones que no vienen de nada y no llevan a nada. Se llevan a cabo y luego no dejan rastro. A menudo son la ilusión de alguien, generalmente algún profesional con ideas "luminosas", que la plantea al margen de su interés general. Al terminarlas uno se da cuenta que se han quemado unas energías y un dinero para nada.

\section{- Proyectos "paracaidistas".}

Son aquellas intervenciones que alguien, generalmente desde la dirección, impone por conveniencia suya, por compromiso institucional, por necesidad estratégica, etc. y que no encajan nada en nuestra dinámica de trabajo. Al profesional le caen de "arriba" y los desarrolla de la mejor manera que sabe pero se da cuenta que no se le respeta su seriedad y estructuración en el trabajo. ¿Para qué diseñar a fondo un proyecto si en cualquier momento te cae un "paracaidista" que te estropea todo tu trabajo? 
- En casa del herrero cuchara de palo.

Los gestores culturales son los primeros en predicar la planificación de los proyectos en los colectivos de artistas o las entidades que plantean una propuesta a una institución. Pero, a su vez, son los primeros también en aplicar para ellos mismos los métodos más artesanales, manuales e impropios de un profesional en su trabajo cotidiano.

- Import-export sin fronteras.

Uno de los grandes males de la profesión. Aquello que funciona en casa del vecino se importa tal cual a nuestra casa. Se deslumbra uno con experiencias ajenas y se quieren repetir sin considerar el contexto en el que han sido desarrolladas. De la misma manera se empeña uno en exportar aquello que, en su caso, ha funcionado bien. Luego no se entiende como algunas actividades tan exitosas en otro sitio no dan los mismos resultados en casa.

- Proyectos "urbi et orbi".

Un defecto de algunos proyectos, en particular en la administración pública, es la de considerar que se dirigen a "todo el mundo" por igual por querer abarcar al máximo de población. Al no definir exactamente las características específicas de su público, no consideran la oportunidad de adaptarlos a unas necesidades particulares lo que hace que nadie se los sienta como suyos. Al final aquello que es para todos, no es para nadie.

- La pirámide del faraón.

Hay quien hace de un proyecto su pirámide por la que pasará a la posteridad. Su fama y su prestigio, aquello que hizo quedará reflejado en tal o cual proyecto para ejemplo de las generaciones venideras. Los proyectos son entonces más la necesidad de un ego mal alimentado que el proceso de reflexión entorno a una realidad concreta. Sucede entonces que cuando la persona que ha dado la personalidad y el sentido al proyecto se retira, este deja de sustentarse y debe readaptarse a fondo o eliminarse.

- Construimos proyectos a la contra.

Los proyectos culturales deberían tener sentido y fuerza en sí mismos, no por estar ideados en contra de algo. Un proyecto debe expresarse en positivo y no debe responder forzosamente a la resolución de un problema planteado. Creemos en la cultura por lo que significa en ella misma y por lo que ayuda a construir en una sociedad y esto debe verse reflejado en los proyectos.

- Agrafía del sector.

Ya se dijo antes, pero constituye una lacra en el sector. ¿Por qué no se hace un esfuerzo en dejar constancia escrita de lo que se hace? ¿Si otras profesiones lo hacen (diagnósticos médicos, planos arquitectónicos, programas escolares, sentencias judiciales, registros deportivos, etc.) por qué en la gestión de la cultura se es tan ágrafo? Nota: un reconocimiento especial en este sentido hacia los profesionales de museos, archivos y bibliotecas: el esfuerzo es mayor.
- Existe un culto al activismo.

En el sector cultural existe un culto al activismo poco común en otras disciplinas profesionales. Está muy valorado todo aquello que sea "hacer algo", un acto, una acción o una actividad. Esto conlleva a que la dirección espera de los profesionales un activismo que, con el tiempo, ha perdido su razón de ser y las cosas se hacen porque hay que hacerlas sin entrar en más consideraciones. Lo peor es que los profesionales acaban entrando por si mismos en esta dinámica y pierden de vista la finalidad última de su intervención.

- Existe un culto a la improvisación.

En el medio profesional existe la creencia de que el buen gestor cultural es aquel o aquella que tiene los recursos propios necesarios para desenvolverse en cualquier situación, por conflictiva que sea. Improvisar (la antítesis de la planificación) se convierte no sólo en un castigo caído sobre la profesión sino una virtud a saber cultivar. Haciendo un paralelismo cultural, el músico que improvisa bien es el que ya sabe tocar (léase diseñar) bien.

- Optimizar recursos no es tan importante como parece. A veces el esfuerzo de planificar para ahorrar costes en un proyecto que partía con pocos recursos se ve desbaratado por la "aparición" de recursos extras para elementos considerados superfluos. $\bigcirc$ sea, cuando se quiere contar con recursos, estos están, pero para el trabajo habitual se trabaja a menudo en régimen de escasez.

- No interesa aglutinar otros agentes.

En algunos casos el querer implicar otros agentes en el proyecto no es tan interesante como a priori parecería. Puede ser que ciertos grupos o instituciones estén más o menos vetados por motivos ideológicos, de intereses partidistas, por estrategias de posicionamiento, etc.

- No interesa la participación del equipo.

La participación de todos los miembros del equipo en el proyecto interesa poco a la dirección y se centra el proyecto en unas pocas personas "pensantes". No se entiende la necesidad de implicar a los demás componentes.

\section{Propuesta de esquema para el diseño de un proyecto cultural}

Llegados a este punto hace falta proponer un esquema que sirva de guía (ipero no de cuestionario!) para llevar a cabo el diseño de un proyecto cultural.

Ante todo hay que reconocer que existen muchos esquemas de todo tipo, la mayoría, importados de otros sectores de actuación, que se parecen mucho entre sí. La propuesta que viene a continuación recoge las aportaciones de, al menos, cuatro disciplinas:

De la planificación estratégica incorpora la estructura inicial en la que se parte de la finalidad para llegar 
a los objetivos pasando antes por dos grandes tamices: el contexto exterior al proyecto y el análisis interno de la organización gestora.

Del trabajo social vale la pena tomar el esfuerzo en la valoración y estructuración del reconocimiento previo del entorno como base para cualquier intervención.

De la pedagogía destacamos la estructuración en las propuestas (contenidos, objetivos, metodología, programa de actividades) y el esfuerzo en la evaluación de los resultados esperados.

Del marketing destaca el interés que presenta el buen conocimiento de los destinatarios como punto de partida para definir el proyecto.

Antes de presentar la propuesta de esquema vale la pena tener en cuenta las siguientes consideraciones:

El esquema se presenta en tres grandes bloques que corresponden a tres estados mentales diferentes:

I. El del contexto es el momento en el que se analizan todos los elementos que se debe conocer antes de tomar cualquier decisión. Termina en un diagnóstico que lo sintetiza. En algunos proyectos el diagnóstico puede ser el inicio del proyecto y todos los análisis previos ser, en su caso, presentados en los anexos.

II. El del contenido es el momento en que, visto el diagnóstico anterior, se exponen las propuestas concretas que definen el proyecto. Se plantean todas las grandes decisiones del proyecto. Es, por lo tanto, su núcleo duro, la parte más importante y la que bajo ningún concepto se puede delegar su responsabilidad.

III. El de los factores de gestión es la parte más operativa del proyecto. A partir de las grandes propuestas de acción este bloque desarrolla todos los pasos necesarios para su puesta en práctica.

El esquema debe verse como una ayuda en el trabajo, no como un corsé que lo limita. Para ello debe saberse adaptarlo a las características de cada caso. El buen esquema es aquel que puede modificarse. En cualquier momento un apartado puede desaparecer si así se considera oportuno (hay que saber argumentarlo), otro puede aparecer, uno puede dividirse en varios o algunos de ellos juntarse en uno, etc. De la misma manera el orden presentado puede alterarse según las necesidades del proyecto. Por ejemplo: el análisis de los destinatarios puede estar en el primer bloque si estos forman parte del punto de partida del proyecto o estar en el segundo bloque si son una decisión más que se toma para definir el proyecto. El bloque final de la evaluación se desarrolla durante y sobretodo al final de la realización del proyecto. En base a los resultados obtenidos se plantea el siguiente.
ESQUEMA GENERAL

Presentación

Breve resumen

I. BASES CONTEXTUALES DEL PROYECTO

1. FINALIDADES

2. DINÁMICA TERRITORIAL

3. DINÁMICA SECTORIAL

4. ENCUADRE EN EL CONTEXTO DE OTRAS POLÍTICAS

5. ANTECEDENTES - ORIGEN

6. ANÁLISIS DE LA ORGANIZACIÓN GESTORA

\section{Diagnóstico}

II. OBJETO Y CONTENIDO DEL PROYECTO

7. DESCRIPCIÓN Y CONTENIDOS

8. OBJETIVOS Y PREVISIÓN DE EVALUACIÓN

9. LÍNEAS ESTRATÉGICAS

10. ACCIONES

11. DESTINATARIOS

12. MODELO DE GESTIÓN

III. FACTORES DE GESTIÓN DEL PROYECTO

13. PLANIFICACIÓN Y PROGRAMACIÓN

14. FACTORES JURÍDICOS

15. ESTRUCTURA ORGANIZATIVA Y RECURSOS HUMANOS

16. GESTIÓN DE LA IMAGEN, LA COMUNICACIÓN Y LA DIFUSIÓN

17. FACTORES INFRAESTRUCTURALES Y TÉCNICOS

18. FACTORES ECONÓMICOS

19. OTROS FACTORES

IV. EVALUACIÓN

20. PROCESO DE EVALUACIÓN

\section{Bibliografía (si se considera necesaria)}

\section{Algunas preguntas habituales}

\section{- Qué extensión debe tener un proyecto?}

Depende de cada caso (autor, cliente, dimensión, tema, tiempo, etc.). Es como si nos preguntáramos cuantas páginas debe tener un libro. Los hay de poco menos de 20 y también hay enciclopedias de varios volúmenes. Aunque la cifra es muy variable, se recomienda hacer proyectos cortos pero completos y claros para facilitar su lectura. No se trata tanto de eliminar mucha información sino de saber sintetizarla.

- ¿Se puede registrar un proyecto para que nadie lo pueda copiar?

Realmente es muy difícil plantear este tema. Un proyecto puede ser otro con pocos cambios. Además ni es un invento (registro de patentes) ni una obra artística (registro de derechos de autor). Lo mejor en estos casos y ante un interlocutor del que se desconfía, es ir mostrando las ideas y los avances del proyecto poco a poco a medida que se vea su interés y predisposición. Nunca hay que mostrar todas las "cartas" a la vez. Hay que dar la sensación de que se tiene más recursos que los presentados y no mostrar el detalle del proyecto hasta el final, en que ya el encargo está totalmente definido. Al contrario también puede ser un buen sistema mostrar ampliamente el proyecto para que se sepa quien lo ha propuesto y que el usurpador tenga un cierto reparo en apropiárselo vista la difusión previa que se le dado. 